\title{
Superconformal Geometry and String Theory
}

\author{
A. A. Rosly, A. S. Schwarz, and A. A. Voronov \\ Institute of Theoretical and Experimental Physics, Bolshaya Cheremushkinskaya, 25, \\ SU-117259 Moscow, USSR
}

\begin{abstract}
We give a formula for the determinant of the super Laplace operator in a holomorphic hermitian line bundle over a superconformal manifold. This is then used to obtain an expression for the fermion string measure.
\end{abstract}

\section{Introduction}

The main purpose of the present paper is to give an expression for the determinant of the super "Laplace" operator associated with an hermitian holomorphic line bundle over a superconformal manifold [Eq. (61) in Sect. 3]. This formula is then used to obtain an expression for the fermion string measure on the superconformal moduli space. A comparison with [6] shows that, like the bosonic string measure which results from the Mumford form, the fermionic string measure can be also obtained from a superanalog of the Mumford form constructed in [6]. (A somewhat different proof of this statement was given in [3].)

The paper is organized as follows. In Sect. 2 we explain in detail the definition and an explicit construction of the super Mumford form of [6]. In Sect. 3 hermitian holomorphic line bundles over a superconformal manifold and the determinants of associated Laplace operators are analysed.

In what follows we exploit heavily the results of [5] on the geometry of superconformal manifolds. Let us recall here some notation from [5]. If $\mathscr{N}$ is a superconformal manifold then $\omega$ denotes a line bundle, the local sections of which take the form $D f$ for a scalar function $f$ and for the spinor derivative $D=\partial / \partial \zeta$ $+\zeta \partial / \partial z$. The space of holomorphic sections of a holomorphic bundle $L$ over $\mathscr{N}$ is denoted by $\mathscr{A}(L)$. A principal simple zero $Q$ of a local holomorphic section $s$ of $L$ is defined by the equations $s(Q)=0, D s(Q)=0$ [and $\partial_{z} s(Q) \neq 0$ for $Q$ being a simple zero]. A principal simple pole of a meromorphic section $s$ is defined as a principal simple pole of $s^{-1}$, provided the latter makes sense. If $\varphi$ is a meromorphic section of the bundle $\omega$, then there exists such a multivalued analytic function $f$ that $\varphi=D f$. The shift in the value of $f$ corresponding to a cycle in the underlying manifold is called the period of $\varphi$. The period which corresponds to turning once 
around $P$, a pole of $\varphi$, is called after dividing by $2 \pi i$ the residue of $\varphi$, to be denoted by $\underset{P}{\operatorname{res}} \varphi$. (For ease of notation we shall write in this paper $\underset{P}{\operatorname{res}} \varphi$ instead of the more correct $\underset{P_{\text {red }}}{\operatorname{res}} \varphi$ used in [5].)

\section{A Superanalog of the Mumford Form}

In this section we describe certain constructions which make it possible to find a measure on the superconformal moduli space. This measure possesses obvious analytic properties as we shall see. After that we argue that the same measure arises from fermion string theory.

Let us consider holomorphic line bundles $L, K, M=K \otimes L$ over a superconformal manifold $\mathscr{N}$ and a holomorphic section $s$ of $K$. Our starting point is a relation between the spaces of holomorphic sections $\mathscr{A}(L), \mathscr{A}\left(L^{*} \otimes \omega\right)$, and the spaces $\mathscr{A}(M), \mathscr{A}\left(M^{*} \otimes \omega\right)$. In other words, we are going to describe a relation between the cohomology groups $H^{0}(\mathscr{N}, L)=\mathscr{A}(L), H^{1}(\mathscr{N}, L)=\Pi\left(\mathscr{A}\left(L^{*} \otimes \omega\right)\right)^{*}$, $H^{0}(\mathscr{N}, M)=\mathscr{A}(M)$, and $H^{1}(\mathscr{N}, M)=\Pi\left(\mathscr{A}\left(M^{*} \otimes \omega\right)\right)^{*}$. We suppose that the section $s_{\text {red }}$ of $K_{\text {red }}$ possesses only simple zeros and denote the corresponding principal zeros of $s$ by $Q_{1}, \ldots, Q_{N}$. Let $M_{i}$ denote the fibre of $M$ over $Q_{i}$ and $M_{i}^{\prime}$ denote the fibre of $M \otimes \omega$ over $Q_{i}$. Both $M_{i}$ and $M_{i}^{\prime}$ are of dimension (1|0). To every local section of $M$ holomorphic near $Q_{i}$ one can assign a point in a (1|1)-dimensional space $W_{i}$ with coordinates $\left(\varphi\left(Q_{i}\right), D \varphi\left(Q_{i}\right)\right)$. The direct sum $W=\otimes W_{i}$ is thus an $(N \mid N)$-dimensional space. Note that the space $\Pi M_{i}^{\prime}$ is embedded naturally in $W_{i}$. The corresponding coset space can be identified with $M_{i}$ :

$$
M_{i}=W / \Pi M_{i}^{\prime} .
$$

These identifications do not depend on coordinates used near $Q_{i}$. Indeed, local coordinates and a local trivialization of $M$ yield coordinates on $W_{i},\left(\varphi\left(Q_{i}\right), D \varphi\left(Q_{i}\right)\right)$ $=(\alpha, \alpha)$, which transform as $(\tilde{\alpha}, \tilde{\alpha})=(g \alpha, F[g \alpha+D g \cdot \alpha])$ under a change of the trivialization described by a function $g$ and a change of coordinates giving $\widetilde{D}=F D$. Identifying $\Pi M_{i}^{\prime}$ with the subspace consisting of points $(0, \alpha)$ in $W_{i}$ one obtains Eq. (1).

The space $H^{0}(\mathscr{N}, L)$ can be embedded in $H^{0}(\mathscr{N}, M)$ by mapping a section $\psi \in H^{0}(\mathscr{N}, L)$ to $\varphi=s \psi \in H^{0}(\mathcal{N}, M)$. The image of this map in $H^{0}(\mathscr{N}, M)$ equals the kernel of another natural map $H^{0}(\mathscr{N}, M) \rightarrow W$. We obtain thus an embedding $\alpha$ of the coset space

$$
V=H^{0}(\mathcal{N}, M) / H^{0}(\mathcal{N}, L)
$$

into $W$. The multiplication by $s$ defines similarly an embedding of $H^{0}\left(\mathscr{N}, M^{*} \otimes \omega\right)$ in $H^{0}\left(\mathscr{N}, L^{*} \otimes \omega\right)$ by virtue of $L^{*} \otimes \omega=K \otimes M^{*} \otimes \omega$. This gives us in the same way as above an embedding $\beta$ of the coset space

$$
V^{\prime}=H^{0}\left(\mathscr{N}, L^{*} \otimes \omega\right) / H^{0}\left(\mathscr{N}, M^{*} \otimes \omega\right)
$$

into $U=\otimes U_{i}$, where

$$
R_{i}=U_{i} / \Pi R_{i}^{\prime}
$$

and $R_{i}, R_{i}^{\prime}$ denote respectively the fibres of $L^{*} \otimes \omega, L^{*} \otimes \omega \otimes \omega$ over the point $Q_{i}$. 
Let us show that

$$
\Pi U_{i}=W_{i}^{*}
$$

for $i=1, \ldots, N$. In order to prove this we have to describe a non-degenerate coupling between the spaces $W_{i}$ and $\Pi U_{i}$. Every element $x$ in $W_{i}$ can be represented as $\left(m\left(Q_{i}\right), \operatorname{Dm}\left(Q_{i}\right)\right)$ for a local holomorphic section $m$ of $M$. Let $y \in U_{i}$ be similarly represented as $\left(r\left(Q_{i}\right), \operatorname{Dr}\left(Q_{i}\right)\right)$ for a local holomorphic section $r$ of $L^{*} \otimes \omega$. Then the coupling between $x$ and $y$ can be defined as follows:

$$
\langle x, y\rangle=\underset{Q_{i}}{\operatorname{res}} \frac{m r}{s} .
$$

This can be written also (using the definition of a residue, see [5]) as

$$
\langle x, y\rangle=D(m r / s)\left(Q_{i}\right),
$$

provided $s=\left(z-z_{i}-\zeta \zeta_{i}\right) \cdot \tilde{s}(z, \zeta)$ near $Q_{i}=\left(z_{i}, \zeta_{i}\right)$. The non-degenerate coupling between $W=\oplus W_{i}$ and $\Pi U=\oplus \Pi U_{i}$ is defined as the sum of residues of the form (4).

Now we are able to define a map $\beta^{*} \lambda$ of $W$ onto $\Pi V^{*}$, where $\lambda$ is the isomorphism between $W$ and $\Pi U^{*}$. One can readily verify that $\beta^{*} \lambda \alpha=0$. For this aim one has to check that the coupling between $x=\alpha x_{1}$ and $y=\beta y_{1}$ gives zero. Indeed, the elements $x \in W$ and $y \in U$ can be represented in this case by global holomorphic sections $m \in H^{0}(\mathscr{N}, M)$ and $r \in H^{0}\left(\mathscr{N}, L^{*} \otimes \omega\right)$. [In fact $m$ is to be chosen from the class $x_{1} \in V=H^{0}(\mathscr{N}, M) / H^{0}(\mathscr{N}, L)$, and similarly $r$ must belong to the class $y_{1} \in V^{\prime}$.] Now $m r / s$ represents a holomorphic field of type $(1 / 2,0)$ which has its poles only over the points $Q_{i \text { red }}$, while the sum of all the residues of such a field is known to be zero. This shows that the map $\beta^{*} \lambda$ defines a map of the coset space $W / V$ in $\Pi V^{\prime *}$. The latter one is in fact an isomorphism. This can be verified by counting the dimensions according to the Riemann-Roch theorem. ${ }^{1}$

We are going to deal with measures on the cohomology spaces. A measure $\mu$ on a complex linear superspace $E$ is understood as a function of the basis $e=\left(e_{1}, \ldots, e_{n}\right)$ in $E$ which obeys $\mu\left(e^{\prime}\right)=\mu(e) \cdot \operatorname{det} C$ for a change of the basis $e_{i}^{\prime}=C_{i}^{j} e_{j}$. Every choice of a basis in $E$ defines obviously a corresponding measure on $E$ demanding that this measure equals 1 on the basis chosen. Any two measures differ by a scalar factor; that is to say, the space of measures, $m_{\mathbb{C}}(E)$, is a one dimensional complex space. It is easy to see that

$$
\begin{aligned}
& m_{\mathbb{C}}\left(E^{*}\right)=m_{\mathbb{C}}(E)^{*}, \\
& m_{\mathbb{C}}(\Pi E)=m_{\mathbb{C}}(E)^{*},
\end{aligned}
$$

\footnotetext{
${ }^{1}$ The isomorphism $W / V=\Pi V^{*}$ is rather easy to prove directly. It follows from a long exact cohomology sequence corresponding to a short exact sequence of sheaves, $0 \rightarrow L \stackrel{s}{\longrightarrow} M \rightarrow \bar{M} \rightarrow 0$, where the map $L \stackrel{s}{\longrightarrow} M$ (embedding of sheaves) is determined by the multiplication by $s$, a holomorphic section of $K=M \otimes L^{*}$; denotes the sheaf corresponding to $M$ restricted to the zero set of $s$, so that, in particular, $H^{0}(\mathcal{N}, \bar{M})=W$ and $H^{1}(\mathcal{N}, \bar{M})=0$. The above short exact sequence gives us $0 \rightarrow H^{0}(\mathcal{N}, L) \rightarrow H^{0}(\mathscr{N}, M) \rightarrow W \rightarrow H^{1}(\mathcal{N}, L) \rightarrow H^{1}(\mathcal{N}, M) \rightarrow 0$ from which the isomorphism $W / V=\Pi V^{*}$ follows immediately by virtue of Serre's duality $H^{1}(\mathscr{N}, L)=\Pi H^{0}\left(\mathscr{N}, L^{*} \otimes \omega\right)^{*}$. Moreover, the Riemann-Roch theorem itself can be proved easily in this way
} 
and, for a (1|0)-dimensional space $E$,

$$
m_{\mathbb{C}}(E)=E^{*}, \quad \operatorname{dim}_{\mathbb{C}} E=1 \mid 0 .
$$

An important role will be played in what follows by the space $m_{\mathbb{C}}\left(\Sigma_{L}\right)$, where

$$
\Sigma_{L}=H^{0}(\mathcal{N}, L) \oplus \Pi H^{0}\left(\mathscr{N}, L^{*} \otimes \omega\right) .
$$

Note that $\Sigma_{L^{*} \otimes \omega}=\Pi \Sigma_{L}$, and hence, by virtue of (7),

$$
m_{\mathbb{C}}\left(\Sigma_{L^{*} \otimes \omega}\right)=m_{\mathbb{C}}\left(\Sigma_{L}\right)^{*} .
$$

If $E^{\prime \prime}=E / E^{\prime}$, then any two given measures on $E^{\prime}$ and $E^{\prime \prime}$ define uniquely a measure on $E$, and one has thus an isomorphism

$$
m_{\mathbb{C}}\left(E^{\prime}\right) \otimes m_{\mathbb{C}}\left(E^{\prime \prime}\right)=m_{\mathbb{C}}(E) .
$$

In order to describe this explicitly let $e=\left(e_{1}, \ldots, e_{r}, e_{r+1}, \ldots, e_{r+q}\right)$ be such a basis in $E$ that $e_{1}, \ldots, e_{r}$ make up a basis in its subspace $E^{\prime}$. If $\pi: E \rightarrow E^{\prime \prime}$ is the natural projection then the vectors $\pi e_{r+1}, \ldots, \pi e_{r+q}$ form a basis in $E^{\prime \prime}$. Given some measures $\mu^{\prime}$ and $\mu^{\prime \prime}$ on respectively $E^{\prime}$ and $E^{\prime \prime}$, the measure $\mu^{\prime} \otimes \mu^{\prime \prime}$ on $E$ is defined by the relation

$$
\left(\mu^{\prime} \otimes \mu^{\prime \prime}\right)(e)=\mu^{\prime}\left(e_{1}, \ldots, e_{r}\right) \cdot \mu^{\prime \prime}\left(\pi e_{r+1}, \ldots, \pi e_{r+q}\right) .
$$

If now some arbitrary basis $\left(e_{1}, \ldots, e_{r+q}\right),\left(e_{1}^{\prime}, \ldots, e_{r}^{\prime}\right)$, and $\left(e_{1}^{\prime \prime}, \ldots, e_{q}^{\prime \prime}\right)$ are chosen in $E$, $E^{\prime}$, and $E^{\prime \prime}$ respectively and $\mu, \mu^{\prime}$, and $\mu^{\prime \prime}$ are the corresponding measures [so that $\mu\left(e_{1}, \ldots, e_{r+q}\right)=1$, etc. $]$ then $\mu$ differs from $\mu^{\prime} \otimes \mu^{\prime \prime}$ by the following factor

$$
\mu=\operatorname{det}\left(\begin{array}{c}
A \\
B^{-1}
\end{array}\right) \cdot \mu^{\prime} \otimes \mu^{\prime \prime},
$$

where the rectangular matrices $A=\left(a_{i j}\right)$ and $B=\left(b_{j k}\right)$ are determined from

$$
\begin{aligned}
e_{i}^{\prime} & =\sum_{j} a_{i j} \cdot e_{j}, \quad i=1, \ldots, r ; j=1, \ldots, r+q, \\
\pi e_{j} & =\sum_{k} b_{j k} \cdot e_{k}^{\prime \prime}, \quad k=1, \ldots, q ; j=1, \ldots, r+q .
\end{aligned}
$$

The rectangular matrix $B^{-1}$ of dimension $q \times(r+q)$ in Eq. (12) is defined as an arbitrary one satisfying $B^{-1} B=I_{q}$.

Equations (11), (6), (7) give us

$$
\begin{gathered}
m_{\mathbb{C}}(V) \equiv m_{\mathbb{C}}\left(H^{0}(\mathscr{N}, M) / H^{0}(\mathscr{N}, L)\right)=m_{\mathbb{C}}\left(H^{0}(\mathscr{N}, M)\right) \otimes m_{\mathbb{C}}\left(H^{0}(\mathscr{N}, L)\right)^{*} \\
m_{\mathbb{C}}\left(V^{\prime}\right) \equiv m_{\mathbb{C}}\left(H^{0}\left(\mathscr{N}, L^{*} \otimes \omega\right) / H^{0}\left(\mathscr{N}, M^{*} \otimes \omega\right)\right) \\
=m_{\mathbb{C}}\left(H^{0}\left(\mathscr{N}, L^{*} \otimes \omega\right)\right) \otimes m_{\mathbb{C}}\left(H^{0}\left(\mathscr{N}, M^{*} \otimes \omega\right)\right)^{*}
\end{gathered}
$$

and, by virtue of the isomorphism $W / V=\Pi V^{\prime *}$,

$$
m_{\mathbb{C}}\left(V^{\prime}\right)=m_{\mathbb{C}}\left(\Pi V^{\prime}\right)^{*}=m_{\mathbb{C}}(W / V)=m_{\mathbb{C}}(W) \otimes m_{\mathbb{C}}(V)^{*}
$$

Using Eqs. (15)-(17) and (9), (10), we obtain

$$
m_{\mathbb{C}}(W)=m_{\mathbb{C}}\left(\Sigma_{L}\right)^{*} \otimes m_{\mathbb{C}}\left(\Sigma_{M}\right) .
$$


Note finally that $m_{\mathbb{C}}(W)=\sigma_{s}$, where $\sigma_{s}$ is the tensor product of the fibres $\omega_{i}$ of the bundle $\omega$ at the points $Q_{i}$,

$$
m_{\mathbb{C}}(W)=\sigma_{s}=\bigoplus_{i} \omega_{i}
$$

This follows from Eqs. (1), (8), (11) (recalling the definition $M_{i}^{\prime}=M_{i} \otimes \omega_{i}$ ); indeed,

$$
\begin{aligned}
m_{\mathbb{C}}\left(W_{i}\right) & =m_{\mathbb{C}}\left(M_{i}\right) \otimes m_{\mathbb{C}}\left(\Pi M_{i}^{\prime}\right)=m_{\mathbb{C}}\left(M_{i}\right) \otimes m_{\mathbb{C}}\left(M_{i}^{\prime}\right)^{*} \\
& =m_{\mathbb{C}}\left(M_{i}\right) \otimes m_{\mathbb{C}}\left(M_{i}\right)^{*} \otimes m_{\mathbb{C}}\left(\omega_{i}\right)^{*}=m_{\mathbb{C}}\left(\omega_{i}^{*}\right)=\omega_{i} .
\end{aligned}
$$

Thus we have a canonical isomorphism

$$
\sigma_{s}=m_{\mathbb{C}}\left(\Sigma_{L}\right)^{*} \otimes m_{\mathbb{C}}\left(\Sigma_{M}\right)=m_{\mathbb{C}}\left(\Sigma_{L}\right)^{*} \otimes m_{\mathbb{C}}\left(\Sigma_{L \otimes K}\right) .
$$

This relation remains valid also if $s$ is a meromorphic section of an arbitrary line bundle $K$ with principal simple zeros $Q_{i}$ and poles $P_{j}$, provided the space $\sigma_{s}$ is defined as follows:

$$
\sigma_{s}=\left(\bigotimes_{i} \omega_{Q_{i}}\right) \otimes\left(\bigotimes_{j} \omega_{P_{J}}\right)^{*}
$$

(This fact can be proved by reducing to the particular case considered above. Indeed, a meromorphic section $s$ of $K$ can be represented as $s=s_{1} / s_{2}$ for some holomorphic sections $s_{1}, s_{2}$ of the bundles $K_{1}, K_{2}$, such that $K_{1} \otimes K_{2}^{*}=K$.)

Let us apply the isomorphism (21) to the case of a holomorphically trivial bundle $L$, i.e. $L=\mathcal{O}$; then

$$
\sigma_{s}=m_{\mathbb{C}}\left(\Sigma_{\mathscr{C}}\right)^{*} \otimes m_{\mathbb{C}}\left(\Sigma_{K}\right) .
$$

Combining Eqs. (21) and (23) we obtain

$$
m_{\mathbb{C}}\left(\Sigma_{L \otimes K}\right)=m_{\mathbb{C}}\left(\Sigma_{L}\right) \otimes m_{\mathbb{C}}\left(\Sigma_{K}\right) \otimes m_{\mathbb{C}}\left(\Sigma_{\mathfrak{O}}\right)^{*} .
$$

This can be rewritten, using the notation

$$
\tilde{m}_{\mathbb{C}}\left(\Sigma_{L}\right)=m_{\mathbb{C}}\left(\Sigma_{L}\right) \otimes m_{\mathbb{C}}\left(\Sigma_{\mathcal{O}}\right)^{*},
$$

as

$$
\tilde{m}_{\mathbb{C}}\left(\Sigma_{L \otimes K}\right)=\tilde{m}_{\mathbb{C}}\left(\Sigma_{L}\right) \otimes \tilde{m}_{\mathbb{C}}\left(\Sigma_{K}\right)
$$

The last relation can be applied to the bundles $\omega^{k}$. Setting

$$
\Sigma_{k}=\Sigma_{\omega^{k}}=\mathscr{A}\left(\omega^{k}\right) \oplus \Pi \mathscr{A}\left(\omega^{1-k}\right),
$$

we have thus

$$
\begin{gathered}
\tilde{m}_{\mathbb{C}}\left(\Sigma_{k}\right)=\tilde{m}_{\mathbb{C}}\left(\Sigma_{1}\right)^{k} \\
m_{\mathbb{C}}\left(\Sigma_{k}\right)=m_{\mathbb{C}}\left(\Sigma_{1}\right)^{k} \otimes m_{\mathbb{C}}\left(\Sigma_{\mathcal{O}}\right)^{1-k}=m_{\mathbb{C}}\left(\Sigma_{1}\right)^{2 k-1} .
\end{gathered}
$$

[We exploited here the relation $m_{\mathbb{C}}\left(\Sigma_{1}\right)=m_{\mathbb{C}}\left(\Sigma_{\mathscr{U}}\right) *$ which represents a particular case of Eq. (10).] In the case of $k=3$, Eq. (26) gives us

$$
m_{\mathbb{C}}\left(\Sigma_{3}\right)=m_{\mathbb{C}}\left(\Sigma_{1}\right)^{5} .
$$


The above relations (26), (27) are analogous to relations proved in the bosonic case by D. Mumford. The isomorphism (27) is mediated by a function $\Phi(e, f)$ of weight 1 with respect to $e$, a basis in $\Sigma_{3}$, and of weight -5 with respect to $f$, a basis in $\Sigma_{1}$. [Here, for a function depending on a basis, to have the weight $w$ with respect to it means that this function is multiplied by $\left(\operatorname{det} C^{w}\right)$ if the basis is transformed with a matrix $C$.] The function $\Phi$ can be called the super Mumford form. It is worth noticing that $\Phi$ is holomorphic on the superconformal moduli space (i.e. with respect to changes of the superconformal manifold $\mathscr{N}$ ), provided of course the bases $e$ and $f$ are chosen to depend holomorphically on the moduli. If the genus $g$ of $\mathscr{N}$ is more than 1 , we have $\mathscr{A}\left(\omega^{-2}\right)=0$, and hence $m_{\mathbb{C}}\left(\Sigma_{3}\right)=m_{\mathbb{C}}\left(\mathscr{A}\left(\omega^{3}\right)\right)$. On the other hand, $\Sigma_{1}=\mathscr{A}(\omega) \oplus \Pi \mathscr{A}(\mathcal{O})$ and, if $\mathscr{N}$ is a normal superconformal manifold [5], $\mathscr{A}(\mathcal{O})$ consists of constant functions, while $\mathscr{A}(\omega)$ is a superspace of dimension $(0 \mid g)$. We are able thus to identify $m_{\mathbb{C}}\left(\Sigma_{1}\right)$ with $m_{\mathbb{C}}(\mathscr{A}(\omega))$. Consequently, Eq. (27) can be written under these circumstances as

$$
m_{\mathbb{C}}\left(\mathscr{A}\left(\omega^{3}\right)\right)=m_{\mathbb{C}}(\mathscr{A}(\omega))^{5} .
$$

Let us consider now real measures on the spaces $\mathscr{A}(\omega), \mathscr{A}\left(\omega^{3}\right)$. We shall denote by $m_{\mathbb{R}}(E)$ the one dimensional real space of real measures on a real linear space $E$. A real measure on $E$ is by definition such a function of the basis in $E$ which after a change of the basis gets multiplied by the modulus of the determinant of the corresponding matrix. If $E$ is a complex linear space we leave the same notation $m_{\mathbb{R}}(E)$ for $E$ considered as a real space. In this case however we can identify $m_{\mathbb{R}}(E)$ with $\left|m_{\mathbb{C}}(E)\right|^{2}$, where the symbol $|F|^{2}$ denotes the subspace of $F \otimes \bar{F}$ consisting of elements invariant under complex conjugation. With these conventions we obtain from Eq. (28),

$$
m_{\mathbb{R}}\left(\mathscr{A}\left(\omega^{3}\right)\right)=m_{\mathbb{R}}(\mathscr{A}(\omega))^{5} .
$$

Let us recall now that there is a natural scalar product in $\mathscr{A}(\omega)$ which is nondegenerate if $\mathscr{N}$ is normal [5]. The scalar product yields a real measure ${ }^{2}$ on $\mathscr{A}(\omega)$. This defines through Eq. (29) an element $d \mu \in m_{\mathbb{R}}\left(\mathscr{A}\left(\omega^{3}\right)\right)$. The latter is however nothing but a real measure on the superconformal moduli space $\mathscr{M}_{g}$. Indeed, $\Pi \mathscr{A}\left(\omega^{3}\right)$ can be identified with the cotangent space to $\mathscr{M}_{g}$ (see Sect. 3 of [5]). On the other hand, to define a measure on the manifold $\mathscr{M}_{g}$ means by definition to define a measure on each tangent space $\mathscr{T}_{\mathscr{N}}\left(\mathscr{M}_{g}\right)$, whereas

$$
m_{\mathbb{R}}\left(\mathscr{T}_{\mathscr{N}}\left(\mathscr{M}_{g}\right)\right)=m_{\mathbb{R}}\left(\mathscr{T}_{\mathscr{N}}^{*}\left(\mathscr{M}_{g}\right)\right)^{*}=m_{\mathbb{R}}\left(\Pi \mathscr{A}\left(\omega^{3}\right)\right)^{*}=m_{\mathbb{R}}\left(\mathscr{A}\left(\omega^{3}\right)\right)
$$

To be more precise, we have thus constructed a measure on a part, $\mathscr{M}_{g}^{\prime}$, of $\mathscr{M}_{g}$ only, where $\mathscr{M}_{g}^{\prime}$ consists of classes of normal superconformal manifolds. Note also that by construction this measure $d \mu$ on $\mathscr{M}_{g}^{\prime}$ can be expressed through the super Mumford form defined in Eq. (27) [or, equivalently, in Eq. (28)]. Namely, if $e$ is a (complex) basis in $\mathscr{A}\left(\omega^{3}\right)=\Sigma_{3}$, then the real measure $d \mu$ takes for this basis the value equal to

$$
d \mu(e, \bar{e})=|\Phi(e, \hat{f})|^{2}=|\Phi(e, f)|^{2}(\operatorname{det} \operatorname{Im} \tau)^{-5},
$$

\footnotetext{
${ }^{2}$ If $\left(e_{i}\right)$ is a complex basis in a complex space $E$ provided with an hermitian scalar product $\langle$,$\rangle ,$ then the real measure corresponding to this scalar product is defined as $\operatorname{det}\left\langle e_{i}, e_{j}\right\rangle$. (In other words, this measure can be determined by any orthonormal frame)
} 
where $\hat{f}$ is an orthonormal basis in $\mathscr{A}(\omega)=\Sigma_{1}$ (note that the left-hand side does not in fact depend on the choice of such a basis); $f=\left(f_{1}, \ldots, f_{q}\right)$ is a basis in $\mathscr{A}(\omega)$ which consists of holomorphic type $(1 / 2,0)$ fields on $\mathscr{N}$ with $A$-periods obeying $A_{i}\left(f_{j}\right)=\delta_{i j}$, while $\tau$ is the corresponding matrix of $B$-periods, $\tau_{i j}=B_{i}\left(f_{j}\right)$. The function $\Phi(e, f)$ is holomorphic in moduli (provided $e$ depends hollomorphically on moduli). Equation (30) describes thus analytic properties of the measure on $\mathscr{M}_{g}^{\prime}$. This measure arises in fact in fermionic string theory. This will be discussed in the next section.

Now we are going to express the measure defined above in terms of certain analytic fields and their zeros on superconformal manifolds. As a matter of fact, all the work is already done. We have only to express a number of the above canonical isomorphisms in terms of explicit formulas.

Let us consider first the canonical isomorphism (21). Let $\beta_{1}, \ldots, \beta_{n}$ be a basis in $\mathscr{A}(L)$ and let $\gamma_{1}, \ldots, \gamma_{k}$ be a basis in $\Pi \mathscr{A}\left(M^{*} \otimes \omega\right)$. In $\mathscr{A}(M)$ we can choose a basis of the form $\left(s \beta_{1}, \ldots, s \beta_{n}, \alpha_{1}, \ldots, \alpha_{m}\right)$, while in $\Pi \mathscr{A}\left(L^{*} \otimes \omega\right)$ we choose a basis $\left(s \gamma_{1}, \ldots, s \gamma_{k}, \varepsilon_{1}, \ldots, \varepsilon_{l}\right)$. If $s$ is an even holomorphic section of $K=M \otimes L^{*}$ and $\sigma$ is an even meromorphic section (with $\sigma_{\text {red }}$ 丰) of $\omega$ [i.e. a field of type $(1 / 2,0)$ ], then let us define $R_{L, M}(s, \sigma)$ as follows. We assume as usual that $s_{\text {red }}$ possesses only simple zeros and $\sigma_{\text {red }} \neq 0, \infty$ at those points. Note that in practice it may be inconvenient to deal only with sections possessing only simple zeros. However the formulas below can be generalized easily to the case of multiple zeros. Let us pick a coordinate system $\left(z^{(i)}, \zeta^{(i)}\right)$ in a neighborhood of each principal simple zero $Q_{i}$, $i=1, \ldots, N$, of $s$ with the origin at $Q_{i}$ in such a way that $\sigma\left(Q_{i}\right)=1$ in these coordinates. Then set

$$
R_{L, M}(S, \sigma)=\operatorname{det}\left(\begin{array}{c}
A \\
B^{-1}
\end{array}\right),
$$

where

$$
\begin{aligned}
& A=\left(\begin{array}{cccc}
\alpha_{1}\left(Q_{1}\right) \ldots \alpha_{1}\left(Q_{N}\right) & D \alpha_{1}\left(Q_{1}\right) \ldots D \alpha_{1}\left(Q_{N}\right) \\
\vdots & \vdots & \vdots & \vdots \\
\alpha_{m}\left(Q_{1}\right) \ldots \alpha_{m}\left(Q_{N}\right) & D \alpha_{m}\left(Q_{1}\right) \ldots D \alpha_{m}\left(Q_{N}\right)
\end{array}\right)
\end{aligned}
$$

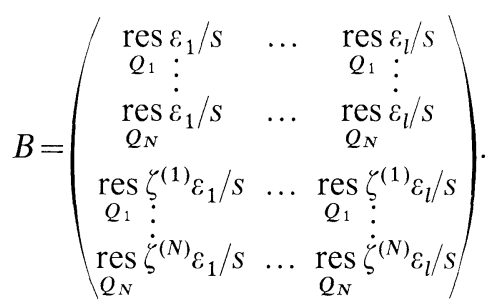

Note that if $s$ is represented near each point $Q_{i}$ as $s=z^{(i)} \cdot \widetilde{s}^{(i)}$ for a non-vanishing function $\widetilde{s}^{(i)}\left(z^{(i)}, \zeta^{(i)}\right)$, then the residues in Eq. (33) can be written as follows:

$$
\begin{gathered}
\underset{Q_{i}}{\operatorname{res}} \varepsilon_{j} / s=D\left(\varepsilon_{j} / \widetilde{S}^{(i)}\right)(0,0), \\
\operatorname{res}_{Q_{i}} \zeta^{(i)} \varepsilon_{j} / s=\varepsilon_{j}(0,0) / \widetilde{s}^{(i)}(0,0) .
\end{gathered}
$$


[We have not indicated local trivializations of the bundles involved which must be chosen of course in a way consistent with the relation $K=M \otimes L^{*}$ and with trivializations of $\omega$ provided by the coordinates $\left(z^{(i)}, \zeta^{(i)}\right)$.] Note also that the matrix on the right-hand side of Eq. (31) is in fact a square one of dimension $(N \mid N)$ $\times(N \mid N)$, for $m+l=N \mid N$ by virtue of the Riemann-Roch theorem.

Every choice of bases in $\mathscr{A}(L), \mathscr{A}\left(L^{*} \otimes \omega\right), \mathscr{A}(M)$, and $\mathscr{A}\left(M^{*} \otimes \omega\right)$ defines certain measures in $\Sigma_{L}$ and $\Sigma_{M}$, and hence an element $v$ in $m_{\mathbb{C}}\left(\Sigma_{L}\right)^{*} \otimes m_{\mathbb{C}}\left(\Sigma_{M}\right)$. On the other hand, every meromorphic section $\sigma$ of $\omega$ defines an element $v^{\prime}$ in $\sigma_{s}$. It can be verified directly that $v^{\prime}$ is mapped by the isomorphism (21) to $R_{L, M}(s, \sigma) \cdot v$, i.e.

$$
v^{\prime}=R_{L, M}(s, \sigma) \cdot v .
$$

In order to prove this one has to note that the vectors $\alpha_{1}, \ldots, \alpha_{m} \in \mathscr{A}(M)$ generate a basis in $V=\mathscr{A}(M) / \mathscr{A}(L)$, the vectors $\varepsilon_{1}, \ldots, \varepsilon_{l} \in \Pi \mathscr{A}\left(L^{*} \otimes \omega\right)$ generate a basis in $\Pi V^{\prime}=\Pi \mathscr{A}\left(L^{*} \otimes \omega\right) / \Pi \mathscr{A}\left(M^{*} \otimes \omega\right)$, and use then Eqs. (12), (31)-(33).

We have thus proved that if $K$ possesses a holomorphic section $s$ the isomorphism (21) can be described by the function $R_{L, M}(s, \sigma)$. The isomorphism (21) corresponding to a meromorphic section $s$ is described by a function which is also denoted by $R_{L, M}(s, \sigma)$ and can be defined in this case as follows:

$$
R_{L, M}(s, \sigma)=R_{L, L \otimes K_{1}}\left(s_{1}, \sigma\right) / R_{M, L \otimes K_{1}}\left(s_{2}, \sigma\right) .
$$

Here $\sigma$ is an even meromorphic section $\left(\sigma_{\text {red }} \neq 0\right)$ of $\omega$ such that $\sigma_{\text {red }} \neq 0, \infty$ at zeros and poles of $s_{\text {red }} ; s_{1}$ and $s_{2}$ are holomorphic sections of bundles $K_{1}$ and $K_{2}$ chosen in such a way that $K=K_{1} \otimes K_{2}^{*}, s=s_{1} / s_{2}$. [Note that the computation of the righthand side of Eq. (37) requires a choice of basis in $\mathscr{A}\left(L \otimes K_{1}\right)$ and $\mathscr{A}\left(L^{*} \otimes K_{1}^{*} \otimes \omega\right)$ which must satisfy an obvious constraint following from the construction. The result is however independent of this choice.]

Proceeding now to Eq. (25), we recall that it is constructed by means of two isomorphisms of the form (21) [namely, Eqs. (21) and (23)]. Consequently, the isomorphism (25) is served by the function

$$
R_{\mathcal{O}, K}(s, \sigma) / R_{L, L \otimes K}(S, \sigma) .
$$

We are able now to compute the Mumford form $\Phi$ which realizes the isomorphism (27). Recalling that the latter isomorphism results from repeated use of $(25)$, we find

$$
\Phi=\frac{R_{\omega^{2}, \omega^{3}}(s, \sigma) R_{\omega, \omega^{2}}(s, \sigma)}{\left[R_{\mathcal{O}, \omega}(s, \sigma)\right]^{2}},
$$

$s$ and $\sigma$ being even meromorphic sections of $\omega$. This expression can be regarded as a superanalog of the Beilinson-Manin formulas in [2]. It must be pointed out that Eq. (39) has a drawback. Indeed, it makes sense only if $\mathscr{A}\left(\omega^{2}\right)$ is a superspace, but this is generally not the case. (This was pointed out to us by A. M. Levin.) However, it is not hard, at the cost of certain complications, to obtain an expression involving only $\mathscr{A}(\mathcal{O}), \mathscr{A}(\omega)$, and $\mathscr{A}\left(\omega^{3}\right)$. Here $\mathscr{A}(\mathcal{O})$ and $\mathscr{A}(\omega)$ are superspaces in the considered case of a normal superconformal manifold, while $\mathscr{A}\left(\omega^{k}\right)$ is always a superspace if $k \geqq 3$. Let $\sigma, s_{1}, s_{2}$ be even meromorphic sections of $\omega$ such that 
$\sigma_{\text {red }} \neq 0, \infty$ at zeros and poles of $s_{1}, s_{2}$. (As usual we assume that $\sigma_{\text {red }} \neq 0$, etc.) Then $s=s_{1} s_{2}$ is a meromorphic section of $\omega^{2}$. Now one can find

$$
\Phi=\frac{R_{\omega, \omega^{3}}(s, \sigma)}{R_{\mathscr{O}, \omega}\left(s_{1}, \sigma\right) R_{\mathscr{O}, \omega}\left(s_{2}, \sigma\right)}
$$

by applying twice Eq. (21) in the case of $L=\mathcal{O}, K=\omega$ with $\sigma_{s_{1}}, i=1$, 2, in the lefthand side and then applying Eq. (21) for $L=\omega, K=\omega^{2}$ with $\sigma_{s}=\sigma_{s_{1}} \otimes \sigma_{s_{2}}$. [Of course, the bases chosen to define the left-hand side of Eq. (40) must obey certain obvious constraints in order to allow for the above threefold use of Eq. (21).]

Equations (30), (39), (40) give us an expression for the measure $d \mu$ on the superconformal moduli space $\mathscr{M}_{g}$, or, more precisely, on its part $\mathscr{M}_{g}^{\prime}$ corresponding to normal superconformal manifolds. The fermion string partition function represents an integral of $d \mu$ over $\mathscr{M}_{g}$ (see Sect. 3). An alternative expression for the partition function can be obtained with the help of an extended moduli space of [3]. Namely, consider a manifold $\hat{\mathscr{M}}_{g}$ which is defined as the total space of a bundle with $\mathscr{M}_{g}^{\prime}$ as its base and $\overline{J(\mathscr{N})^{5}}$ as its fibre over the point represented by a superconformal manifold $\mathscr{N}$. Here $J(\mathscr{N})$ is the Jacobian of $\mathscr{N}$ and $\overline{J(\mathscr{N})^{5}}$ deenotes the direct product of five copies of $\overline{J(\mathscr{N})}$, a manifold complex conjugated to $J(\mathscr{N})$. The manifold $J(\mathscr{N})$ can be represented as a quotient of the space $\Pi \bar{\Sigma}_{1}$ over a lattice (see [5]). The tangent space $\mathscr{T} \hat{\mathscr{M}}_{g}$ contains a subspace $5 \Pi \Sigma_{1}$ (the direct sum of five copies of $\left.\Pi \Sigma_{1}\right)$, so that $\mathscr{T} \hat{\mathscr{M}}_{g} / 5 \Pi \Sigma_{1}=\mathscr{T} \mathscr{M}_{g}^{\prime}=\Pi \Sigma_{3}^{*}$. This implies

$$
m_{\mathbb{C}}\left(\mathscr{T} \hat{\mathscr{M}}_{g}\right)=m_{\mathbb{C}}\left(\Pi \Sigma_{3}^{*}\right) \otimes m_{\mathbb{C}}\left(5 \Pi \Sigma_{1}\right)=m_{\mathbb{C}}\left(\Sigma_{3}\right) \otimes m_{\mathbb{C}}\left(\Sigma_{1}\right)^{-5}=\mathbb{C},
$$

where the last equality is due to Eq. (27). The canonical isomorphism $m_{\mathbb{C}}\left(\mathscr{T} \hat{\mathscr{M}}_{g}\right)=\mathbb{C}$ defines a holomorphic measure $\hat{\Phi}$ on $\hat{\mathscr{M}}_{g}$. (As a matter of course, $\hat{\Phi}$ is nothing but the pull-back of $\Phi$ to $\hat{\mathscr{M}}_{g}$.) Since the volume of $J(\mathscr{N})$ equals 1 with respect to the natural metric on the Jacobian, it follows that the integral of the modulus squared of $\hat{\Phi}$ over $\hat{\mathscr{M}}_{g}$ coincides with the integral of $d \mu$ over $\mathscr{M}_{g}^{\prime}$, giving thus the same partition function.

\section{Hermitian Holomorphic Line Bundles. Determinants of Laplace Operators}

Let us consider an hermitian holomorphic line bundle $L$ over a superconformal manifold $\mathscr{N}$. (That is to say, we assume that an hermitian scalar product is defined in the fibres of $L$.) The field $F_{L}$ of type $(1 / 2,1 / 2)$ defined via

$$
F_{L}=D \bar{D} \log \|s\|_{L}
$$

will be called the curvature of the hermitian bundle $L$. Here $s$ is an arbitrary local even holomorphic section of $L$ (with $s_{\text {red }} \neq 0$ ) and $F_{L}$ is obviously independent of the choice of $s .^{3}$

\footnotetext{
${ }^{3}$ The curvature of an hermitian holomorphic bundle over an arbitrary complex supermanfiold can be defined also as a $(1,1)$ differential form in the usual way. In the case of a superconformal manifold two definitions of the curvature are related by means of the map $\beta$ described in Sect. 1 of [5]
} 
An hermitian structure in the bundle $\omega$ is referred to as a super Riemann metric on a superconformal manifold. (This definition agrees with that given in [3].) The curvature of the hermitian structure in $\omega$ will be called the curvature of the super Riemann metric. Let us denote it by $R$; thus

$$
R=D \bar{D} \log \|\varphi\|_{\omega}
$$

where $\varphi$ is a local even holomorphic field of type $(1 / 2,0)$.

In a manifold endowed with a super Riemann metric, there is a natural supervolume element $d v$ (cf. [3]). This allows one to introduce a scalar product in the space $\Gamma(L)$ of sections of an hermitian bundle $L$; namely

$$
\left\langle\varphi_{1}, \varphi_{2}\right\rangle_{L}=\int_{\mathscr{N}}\left(\varphi_{1}, \varphi_{2}\right)_{L} d v
$$

with $\left(\varphi_{1}, \varphi_{2}\right)_{L}$ being the scalar product in the fibre. By means of scalar products in $\Gamma(L)$ and $\Gamma(L \otimes \bar{\omega})$ one defines an hermitian conjugated operator $\bar{D}_{L}^{+}$for the $\bar{D}$-operator associated with a holomorphic structure of $L, \bar{D}_{L}: \Gamma(L) \rightarrow \Gamma(L \otimes \bar{\omega})$. Then the operator

$$
\square_{L}=\bar{D}_{L}^{+} \bar{D}_{L}
$$

acts in the space $\Gamma(L)$. Our aim is to study the determinant (i.e. Berezinian) of $\square_{L}$. This will allow us to work out the string measure on the superconformal moduli space. (This measure can be written in terms of $\operatorname{det} \square_{\omega}$ and $\operatorname{det} \square_{\omega^{3}}$.)

The determinant det $\square_{L}$ can be conveniently dealt with in terms of a measure on the space of zero modes. Namely, consider the superspace $\Sigma_{L}=\mathscr{A}(L) \oplus \Pi \mathscr{A}\left(L^{*} \otimes \omega\right)$ constructed from the kernel and the cokernel of $D_{L}$ and take some bases, $\left(\alpha_{1}, \ldots, \alpha_{m}\right)$ in $\mathscr{A}(L)$ and $\left(\beta_{1}, \ldots, \beta_{n}\right)$ in $\Pi \mathscr{A}\left(L^{*} \otimes \omega\right)$. Let us define $\left(N_{L}\right)_{i j}=\left\langle\alpha_{i}, \alpha_{j}\right\rangle,\left(N_{L}^{\prime}\right)_{k l}=\left\langle\beta_{k}, \beta_{l}\right\rangle$ by means of Eq. (44) (we suppress the subscripts $L$ and $L^{*} \otimes \omega$ in $\left.\langle\rangle,\right)$. Then the expression

$$
d \mu_{L}=\left(\operatorname{det} \square_{L}\right)^{-1} \operatorname{det} N_{L} \operatorname{det} N_{L}^{\prime} d^{m} a d^{n} b d^{m} \bar{a} d^{n} \bar{b},
$$

with $a_{i}, b_{k}$ being respectively complex coordinates in $\mathscr{A}(L), \Pi \mathscr{A}\left(L^{*} \otimes \omega\right)$ corresponding to the bases $\alpha, \beta$, can be considered as a real measure on $\Sigma_{L} \cdot{ }^{4} \mathrm{~A}$ variation of $d \mu_{L}$ under an infinitesimal variation of the hermitian metric in $L$ can be expressed in terms of Seeley coefficients. (The variation of det $\square_{L}$ is given by Seeley coefficients and zero modes $\alpha, \beta$, while in the variation of $d \mu_{L}$ the contribution of zero modes cancels [3].) Computing the Seeley coefficients, one finds

$$
\delta d \mu_{L}=\left(\frac{1}{\pi} \int \delta \log \|s\|_{L}^{2} \cdot R d^{2} z d^{2} \zeta\right) \cdot d \mu_{L},
$$

\footnotetext{
${ }^{4}$ Note that the measure $d \mu_{L}$ differs by a factor of $\left(\operatorname{det} \square_{L}\right)^{-1}$ from the measure defined by the scalar product in $\Sigma_{L}$. The super Riemann metric on $\mathscr{N}$ and the metric in the fibres of $L$ can be chosen in such a way that the scalar product (44) restricted to the subspace of holomorphic sections is nondegenerate. (This can be, for instance, the super Poincaré metric on $\mathscr{N}$.) In particular, the super volume $\int_{\mathscr{N}} d v$ of $\mathscr{N}$ will be then an invertible element, i.e. $\left(\int_{\mathscr{V}} d v\right)_{\text {red }} \neq 0$
} 
where $s$ is a meromorphic section of $L$ and $R$ is the curvature of the super Riemann metric on $\mathscr{N}$. In the case of a holomorphically trivial bundle $L$ Eq. (47) implies immediately

$$
d \mu_{L}=d \mu_{\mathscr{O}} \cdot \exp \left(\frac{1}{\pi} \int \log \|s\|_{L}^{2} \cdot R d^{2} z d^{2 \zeta}\right),
$$

where $s$ is now a non-vanishing holomorphic section of $L$, while $\mathcal{O}$ stands for a direct product bundle $\mathscr{N} \times \mathbb{C}^{1 / 0}$ endowed with the standard flat metric. (Notice that $L$ and $\mathcal{O}$ are isomorphic as holomorphic bundles and differ only by a choice of hermitian metric. The measures $d \mu_{L}$ and $d \mu_{\mathscr{O}}$ are defined thus on the same space.) We are going now to generalize Eq. (48) to the case of an arbitrary hermitian holomorphic line bundle. For this purpose we consider additionally certain constructions which are also of an independent importance.

Let $s$ and $s^{\prime}$ be even meromorphic sections of hermitian holomorphic line bundles $L$ and $L^{\prime}$, respectively. These sections are supposed to possess only simple principal zeros and poles. Let $Q_{i}$ and $P_{j}$ be the principal simple zeros and poles of $s$ and let $Q_{k}^{\prime}, P_{l}^{\prime}$ play the same role for $s^{\prime}$. We define then $\left\{s^{\prime}, s\right\}$ by the following relation:

$$
\left\{s^{\prime}, s\right\}=\exp \left[-\frac{1}{\pi} \int F_{L^{\prime}} \log \|s\|_{L}+\sum_{i} \log \left\|s^{\prime}\left(Q_{i}\right)\right\|_{L^{\prime}}-\sum_{j} \log \left\|s^{\prime}\left(P_{j}\right)\right\|_{L^{\prime}}\right] .
$$

It is easy to verify that

$$
\left\{s^{\prime}, s\right\}=\left\{s, s^{\prime}\right\} .
$$

Indeed, $\left\{s^{\prime}, s\right\}$ can be rewritten as

$$
\begin{aligned}
\log \left\{s^{\prime}, s\right\}= & -\frac{1}{\pi} \int \log \|s\|_{L} D \bar{D}\left\|s^{\prime}\right\|_{L^{\prime}}+\sum_{i} \log \left\|s^{\prime}\left(Q_{i}\right)\right\|_{L^{\prime}}-\sum_{j} \log \left\|s^{\prime}\left(P_{j}\right)\right\|_{L^{\prime}} \\
& +\sum_{k} \log \left\|s\left(Q_{k}^{\prime}\right)\right\|_{L}-\sum_{l} \log \left\|s\left(P_{l}^{\prime}\right)\right\|_{L},
\end{aligned}
$$

and the symmetry property (50) follows by integrating by parts. $D \bar{D} \log \left\|s^{\prime}\right\|_{L^{\prime}}$ in Eq. (51) is understood in terms of distributions, so that

$$
D \bar{D} \log \left\|s^{\prime}\right\|_{L^{\prime}}=F_{L^{\prime}}+\pi\left(\sum_{i} \delta_{Q_{\imath}}-\sum_{j} \delta_{P_{J}}\right),
$$

where $\delta_{P}$ denotes a $\delta$-function on $\mathscr{N}$ supported at the point $P$; if $P=\left(z_{0}, \zeta_{0}\right)$ then

$$
\delta_{P}=\delta^{(2)}\left(z-z_{0}\right) \cdot\left(\zeta-\zeta_{0}\right) \cdot\left(\bar{\zeta}-\bar{\zeta}_{0}\right) .
$$

It is easy to find a change of $\left\{s^{\prime}, s\right\}$ caused by a change in $s$ and $s^{\prime}$; by virtue of (49), one has in fact for a meromorphic function $\varphi$

$$
\log \left\{\varphi s^{\prime}, s\right\}=\log \left\{s^{\prime}, s\right\}+\sum_{i} \log \left|\varphi\left(Q_{i}\right)\right|-\sum_{j} \log \left|\varphi\left(P_{j}\right)\right| .
$$

Equation (49) allows us also to express the relation (48) in the following form:

$$
d \mu_{L} / d \mu_{\mathscr{O}}=\{s, \sigma\}^{-2},
$$


where $\sigma$ is a meromorphic section of the bundle $\omega$. The latter relation hints at the form of an analogous relation for $d \mu_{L}$ in case of an arbitrary (holomorphically nontrivial) hermitian bundle $L$, as we shall see shortly. For this aim we have to clarify the invariant meaning of $\left\{s^{\prime}, s\right\}$.

First of all let us consider the following construction. Given any two holomorphic line bundles $L$ and $L^{\prime}$ one can define a one-dimensional complex vector space $\left[L^{\prime}, L\right]$ as follows. If $s$ is as before an even meromorphic section of $L$ with principal simple zeros at $Q_{i}$ and principal simple poles at $P_{j}$, then one defines $\left[L^{\prime}, L\right]=\left(\otimes_{i} L_{Q_{\imath}}^{\prime}\right) \otimes\left(\otimes_{j} L_{P_{\jmath}}^{\prime}\right)^{*} .5$ This definition is in fact correct; $\left[L^{\prime}, L\right]$ does not depend on the choice of $s$. (That is to say, the spaces $\left[L^{\prime}, L\right]$ given by different choices of $s$ are canonically isomorphic, as it will be seen below.) Moreover, one has the following canonical isomorphisms:

$$
\begin{gathered}
{\left[L^{\prime}, L\right]=\left[L, L^{\prime}\right],} \\
{\left[L^{\prime}, L^{*}\right]=\left[L^{\prime}, L\right]^{*},} \\
{\left[L^{\prime}, L_{1} \otimes L_{2}\right]=\left[L^{\prime}, L_{1}\right] \otimes\left[L^{\prime}, L_{2}\right] .}
\end{gathered}
$$

The last two of these are obvious from the definition. The isomorphism (54), as well as the consistency of the definition itself, follows from the symmetry $\left[\varphi^{\prime}, \varphi\right]$ $=\left[\varphi, \varphi^{\prime}\right]$ of the Weil coupling, $\left[\varphi^{\prime}, \varphi\right]$, of two meromorphic functions $\varphi$ and $\varphi^{\prime}$ discussed in Sect. 2 of [5]. Let us define now the Weil coupling $\left[\varphi^{\prime}, s\right]$ of a meromorphic function $\varphi^{\prime}$ and a meromorphic section $s$ of $L$;

$$
\left[\varphi^{\prime}, s\right]=\prod_{i} \varphi^{\prime}\left(Q_{i}\right) / \prod_{j} \varphi^{\prime}\left(P_{j}\right),
$$

with $Q_{i}$ being principal simple zeros and $P_{j}$ being principal simple poles of $s$. [The coupling of two meromorphic functions is of course a particular case of (57). ] Now we are able to give (following Deligne [4]) an alternative definition of $\left[L^{\prime}, L\right]$ which makes the above properties obvious. The one-dimensional complex space $\left[L^{\prime}, L\right]$ is defined as a set of equivalence classes $\left[s^{\prime}, s\right]$, where $s^{\prime}, s$ are even meromorphic sections of $L^{\prime}, L$ which possess only principal simple zeros and poles such that the set of zeros and poles of $s^{\prime}$ does not intersect the same set for $s$. If $\tilde{s}^{\prime}, \tilde{s}$ is another pair of sections and $\varphi^{\prime}=\tilde{s}^{\prime} / s^{\prime}, \varphi=\tilde{s} / s$ are thus meromorphic functions, then we set

$$
\left[\tilde{s}^{\prime}, \tilde{s}\right]=\left[s^{\prime}, s\right] \cdot\left[\varphi^{\prime}, s\right] \cdot\left[\varphi, s^{\prime}\right] \cdot\left[\varphi^{\prime}, \varphi\right],
$$

where the factors $\left[\varphi^{\prime}, s\right],[\varphi, s],\left[\varphi^{\prime}, \varphi\right]$ are already defined by means of Eq. (57). We obtain thus a definition of $\left[L^{\prime}, L\right]$ and of the Weil-Deligne coupling $\left[s^{\prime}, s\right]$ for meromorphic sections, so that $\left[s^{\prime}, s\right] \in\left[L^{\prime}, L\right]$. Now it is straightforward to see that the isomorphism of $\left[L^{\prime}, L\right]$ and $\left[L, L^{\prime}\right]$ which identifies $\left[s^{\prime}, s\right]$ and $\left[s, s^{\prime}\right]$ is well defined by virtue of the symmetry $\left[\varphi^{\prime}, \varphi\right]=\left[\varphi, \varphi^{\prime}\right]$ for functions. It remains to show that the latter definition of $\left[L^{\prime}, L\right]$ coincides with the former. This can be proved by

\footnotetext{
${ }^{5}$ Note that we have already met an example of such a construction. The space $\sigma_{s}$ considered in Sect. 2 with $s$ being there a meromorphic section of a bundle $K$ can be regarded as $[\omega, K]$. Equation (23) can be thus written as $m_{\mathbb{C}}\left(\Sigma_{K}\right) \otimes m_{\mathbb{C}}\left(\Sigma_{\mathscr{G}}\right)^{*}=[\omega, K]$. Note also that this relation together with $m_{\mathbb{C}}\left(\Sigma_{\omega}\right)=m_{\mathbb{\mathbb { C }}}\left(\Sigma_{\mathscr{O}}\right)^{*}[$ cf. Eq. $(10)]$ yields $m_{\mathbb{\mathbb { C }}}\left(\Sigma_{K}\right)^{2}=[\omega, K]^{2} \otimes[\omega, \omega]^{-1}$
} 
noticing that $\left[s^{\prime}, s\right]$ can be identified with the point $\left(\underset{i}{\otimes} s^{\prime}\left(Q_{i}\right)\right) \otimes\left(\underset{j}{\otimes} s^{\prime}\left(P_{j}\right)\right)^{-1}$ in the space $\left(\underset{i}{\otimes} L_{Q_{l}}^{\prime}\right) \otimes\left(\underset{j}{\otimes} L_{P_{J}}^{\prime}\right)^{*}$.

The definition of $\left\{s^{\prime}, s\right\}$ [Eq. (49)] allows us to introduce a norm in the space $\left[L^{\prime}, L\right]$ for hermitian holomorphic bundles $L^{\prime}, L$. If $x=\left[s^{\prime}, s\right] \in\left[L^{\prime}, L\right]$, we set $\|x\|=\left\{s^{\prime}, s\right\}$. One can see directly from Eqs. (52) and (58) that $\|x\|$ depends only on $x \in\left[L^{\prime}, L\right]$ and not on the choice of sections $s^{\prime}, s$ and that $\|\hat{\lambda} x\|=|\lambda| \cdot\|x\|$ with a complex number $\lambda$ as required for the definition of a norm. Moreover, Eqs. (50), (52) show that the isomorphisms (54)-(56) are in fact isometries with respect to this norm. Since the complex space $\left[L^{\prime}, L\right]$ is one-dimensional, one can consider $\|\cdot\|^{2}$ as a real measure on it or, in other words, as an element in $\left|\left[L^{\prime}, L^{*}\right]\right|^{2}$. (Recall that $m_{\mathbb{R}}(E)=\left|m_{\mathbb{d}}(E)\right|^{2}$ and that $m_{\mathbb{d}}\left(\left[L^{\prime}, L\right]\right)=\left[L^{\prime}, L\right]^{*}=\left[L^{\prime}, L^{*}\right]$.) Therefore, to every two hermitian holomorphic line bundles $L^{\prime}$ and $L$ we relate an element in $\left|\left[L^{\prime}, L^{*}\right]\right|^{2}$. Let us denote it by $\kappa\left(L^{\prime}, L^{*}\right) \in\left|\left[L^{\prime}, L^{*}\right]\right|^{2}$.

According to the notation introduced in Eq. (46), $d \mu_{L}$ is a real measure on the complex space $\Sigma_{L}$ and $d \mu_{\mathscr{O}}$ is a real measure on $\Sigma_{\mathscr{O}}$; i.e. $d \mu_{L} \in m_{\mathbb{R}}\left(\Sigma_{L}\right), d \mu_{\mathscr{O}} \in m_{\mathbb{R}}\left(\Sigma_{\mathcal{O}}\right)$. This implies that $d \mu_{L} / d \mu_{\mathscr{O}}$ can be thought of as an element in $m_{\mathbb{R}}\left(\Sigma_{L}\right) \otimes m_{\mathbb{R}}\left(\Sigma_{\mathscr{\Theta}}\right)^{*}$ $=\left|m_{\mathbb{C}}\left(\Sigma_{L}\right) \otimes m_{\mathbb{C}}\left(\Sigma_{\mathcal{O}}\right)^{*}\right|^{2}$. We have proved in Sect. 2 that there exists a canonical isomorphism $\tau$ between $m_{\mathbb{C}}\left(\Sigma_{L}\right) \otimes m_{\mathbb{C}}\left(\Sigma_{\mathbb{G}}\right)^{*}$ and $[\omega, L]$ (see Eq. (23) and the last footnote). Let $|\tau|^{2}$ denote the corresponding isomorphism between $\left|m_{\mathbb{Q}}\left(\Sigma_{L}\right) \otimes m_{\mathbb{C}}\left(\Sigma_{\Theta}\right)^{*}\right|^{2}$ and $|[\omega, L]|^{2}$. This suggests that the proper generalization of Eq. (53) takes the following form:

$$
d \mu_{L} / d \mu_{\varrho}=|\tau|^{2}(\kappa(\omega, L)) .
$$

In the bosonic case such a formula was proved by Deligne [4]. It is easy to check that for a holomorphically trivial bundle $L$ Eq. (59) reduces to (53). On the other hand, Eq. (47) shows that both sides of the relation (59) vary in the same way under a variation of hermitian metric in $L$. A complete proof would follow by verifying that the same remains valid for variations of the holomorphic structure of $L$ (it seems that this can be done using a super-generalization of Quillen's formulas given in [3]) and by checking then Eq. (59) for at least one bundle $L$ on each $\mathscr{N}$.

Applying the explicit form of the isomorphism $\tau$ [i.e. of the isomorphism (23)] we get formulas for $d \mu_{L}$ and $\operatorname{det} \square_{L}$. The result is as follows:

$$
\mathrm{d} \mu_{L}=\left|R_{\mathscr{O}, L}(s, \sigma)\right|^{2}\{s, \sigma\}^{-2} d \mu_{\mathscr{O}},
$$

where $\left|R_{\mathscr{O}, L}(s, \sigma)\right|^{2}$ defined by (31)-(33) is regarded as a function of real bases in $\Sigma_{L}$ and $\Sigma_{\mathscr{O}}$ of weights 1 and -1 respectively. Recalling Eq. (46), we obtain

$$
\operatorname{det} \square_{L}=\frac{\operatorname{det} N_{L} \operatorname{det} N_{L}^{\prime}}{\int_{\mathscr{N}} d v \cdot \operatorname{det} N_{\mathcal{O}}^{\prime}} \cdot\left|R_{\mathcal{U}, L}(s, \sigma)\right|^{-2}\{s, \sigma\}^{2} \operatorname{det} \square_{\mathcal{O}},
$$

where $\operatorname{det} N_{L}, \operatorname{det} N_{L}^{\prime}, \operatorname{det} N_{\mathscr{O}} \equiv \int_{\mathcal{S}} d v$, and $\operatorname{det} N_{\mathscr{O}}^{\prime}$ are the determinants of scalar products of basis vectors in, respectively, $\mathscr{A}(L), \Pi \mathscr{A}\left(L^{*} \otimes \omega\right), \mathscr{A}(\mathcal{O})$, and $\Pi \mathscr{A}(\omega)$. (The "determinants" are always understood as superdeterminants.) Introducing the notation

$$
\widetilde{\operatorname{det}} \square_{L}=\frac{\operatorname{det} \square_{L}}{\operatorname{det} N_{L} \operatorname{det} N_{L}^{\prime}},
$$


we obtain immediately from $(61)^{6}$,

$$
\widetilde{\operatorname{det}} \square_{L \otimes M}=\frac{\widetilde{\operatorname{det}} \square_{L} \widetilde{\operatorname{det}} \square_{M}}{\widetilde{\operatorname{det}} \square_{\mathcal{O}}} \cdot \frac{\left|R_{\mathcal{O}, L}(s, \sigma)\right|^{2}}{\left|R_{M, L \otimes M}(s, \sigma)\right|^{2}} .
$$

The latter relation was derived in [3], where it was used in construction of the fermion string measure. In fact, Eq. (62) yields

$$
\widetilde{\operatorname{det}} \square_{\omega^{3}}\left(\widetilde{\operatorname{det}} \square_{\omega}\right)^{-5}=\frac{\left|R_{\mathscr{O}, \omega}(s \cdot \sigma)\right|^{4}}{\left|R_{\omega^{2}, \omega^{3}}(s, \sigma)\right|^{2} \cdot\left|R_{\omega, \omega^{2}}(s, \sigma)\right|^{2}} .
$$

We have used here (62) with $L=\omega, M=\omega^{2}$ and with $L=M=\omega$ [and the relation $\left.\overline{\operatorname{det}} \square_{\mathcal{O}}=\left(\overline{\operatorname{det}} \square_{\omega}\right)^{-1}\right]$. A quick comparison with Eqs. (39), (30) shows that the fermion string measure $\left(\frac{1}{\operatorname{det}} \square_{\omega^{3}}\right)^{-1} \cdot\left(\operatorname{det} \square_{\mathcal{O}}\right)^{-5}$ does indeed coincide with the real measure constructed in Sect. 2 by means of the super Mumford form. [Note that, as we have pointed out at the end of Sect. 2, the relation (63) is of restricted use. This can be improved with the help of expression (40) for the Mumford form and using an analogous expression for $\widehat{\operatorname{det}} \square_{\omega^{3}} \cdot\left(\operatorname{det} \square_{\omega}\right)^{-5}$.]

Acknowledgements. We are grateful to M. A. Baranov, A. M. Levin, and Yu. I. Manin.

\section{References}

1. Baranov, M.A., Schwarz, A.S.: Multiloop contribution to string theory. Pis'ma v ZhETF 42:8, 340 (1985) [JETP Letters 42, 419 (1986)]

2. Beilinson, A.A., Manin, Yu.I.: The Mumford form and the Polyakov measure in string theory. Commun. Math. Phys. 107, 359 (1986)

3. Baranov, M.A., Schwarz, A.S.: On the multiloop contribution to the string theory. Int. J. Mod. Phys. A 3, 28 (1987)

4. Deligne, P.: Le determinant de la cohomologie. Contemp. Math. 67, 93 (1987)

5. Rosly, A.A., Schwarz, A.S., Voronov, A.A.: Geometry of superconformal manifolds. Commun. Math. Phys. 113, 129-152 (1988)

6. Voronov, A.A.: A formula for the Mumford measure in superstring theory. Funk. Anal. Prilozh. 22, 67 (1988)

Communicated by Ya. G. Sinai

Received May 10, 1988

${ }^{6}$ Rather, one has to use an obvious generalization of Eq. (61), namely,

$$
\widetilde{\operatorname{det}} \square_{L \otimes M}=\widetilde{\operatorname{det}} \square_{M}\left|R_{\mathscr{O}, L}(s, \sigma)\right|^{-2}\{s, \sigma\}^{2}
$$

